\title{
Gut microbiota of mice putatively modifies amino acid metabolism in the host brain
}

\author{
Takahiro Kawase $^{1}$, Mao Nagasawa ${ }^{1}$, Hiromi Ikeda ${ }^{1}$, Shinobu Yasuo ${ }^{1}$, Yasuhiro Koga ${ }^{2}$ \\ and Mitsuhiro Furuse ${ }^{1 *}$ \\ ${ }^{1}$ Laboratory of Regulation in Metabolism and Behavior, Graduate School of Bioresource and Bioenvironmental Sciences, \\ Kyushu University, Fukuoka 812-8581, Japan \\ ${ }^{2}$ Laboratory for Infectious Diseases, School of Medicine, Tokai University, Kanagawa 259-1193, Japan
}

(Submitted 20 August 2016 - Final revision received 14 February 2017 - Accepted 22 February 2017 - First published online 10 April 2017)

\section{Abstract}

Recently, it has been found that the gut microbiota influences functions of the host brain by affecting monoamine metabolism. The present study focused on the relationship between the gut microbiota and the brain amino acids. Specific pathogen-free (SPF) and germ-free (GF) mice were used as experimental models. Plasma and brain regions were sampled from mice at 7 and 16 weeks of age, and analysed for free D- and L-amino acids, which are believed to affect many physiological functions. At 7 weeks of age, plasma concentrations of D-aspartic acid (D-Asp), L-alanine (L-Ala), L-glutamine (L-Gln) and taurine were higher in SPF mice than in GF mice, but no differences were found at 16 weeks of age. Similar patterns were observed for the concentrations of L-Asp in striatum, cerebral cortex and hippocampus, and L-arginine (L-Arg), $\mathrm{L}$-Ala and $\mathrm{L}-\mathrm{valine}(\mathrm{L}-\mathrm{Val}$ ) in striatum. In addition, the concentrations of L-Asp, D-Ala, L-histidine, L-isoleucine (L-Ile), L-leucine (L-Leu), L-phenylalanine and L-Val were significantly higher in plasma of SPF mice when compared with those of GF mice. The concentrations of L-Arg, L-Gln, L-Ile and L-Leu were significantly higher in SPF than in GF mice, but those of D-Asp, D-serine and L-serine were higher in some brain regions of GF mice than in those of SPF mice. In conclusion, the concentration of amino acids in the host brain seems to be dependent on presence of the gut microbiota. Amino acid metabolism in the host brain may be modified by manipulating microbiota communities.

Key words: Gut microbiota: Germ-free mice: D-Amino acids: L-Amino acids: Brain: Ageing

Animals, except for those with a specific condition - namely, germ-free (GF) status - have symbiotic relationships with body bacteria, especially those in their digestive system ${ }^{(1,2)}$. In the host intestine, gut bacteria play many crucial roles, such as developing the postnatal immune system of the host, assisting in the efficiency of digestion and absorption of macromolecules, and acting as a barrier against pathogen attachment to gut binding sites, which is the first step of bacterial pathogenicity ${ }^{(3,4)}$. Moreover, intestinal micro-organisms have been found to affect the central level of the brain-derived neurotropic factor, have a role in anxiety- or depression-like mood disorders and influence the response of the hypothalamic-pituitary-adrenal (HPA) axis in mice during restraint stress ${ }^{(5-7)}$. There have also been indications that the intestinal microbiota is concerned with anxiety-like behaviour and central neurochemical changes in mice ${ }^{(8)}$. Mice which had gut populations of commensal faecal microbiota were found to be less anxious and active, and to have higher noradrenaline (NA), dopamine (DA) and serotonin turnover rates in several brain regions, when compared with GF mice ${ }^{(9)}$.
In addition, there is abundant evidence suggesting that the gut microbiota composition, the concentration of microbial products and gastrointestinal symptoms in humans are closely associated with disorders of the central nervous system (CNS) such as autism spectrum disorder and depression ${ }^{(10-12)}$.

In the brain, amino acids function as neurotransmitters or as substrates of monoamine neurotransmitters and are involved in protein synthesis. Many amino acids and their metabolites in the brain have sedative and hypnotic effects in chicks ${ }^{(13)}$. D-Amino acids are synthesised from L-amino acids by racemase, an isomerase enzyme found in the brain and peripheral regions, and which is also expressed in the microbiota ${ }^{(14-16)}$. A number of functions of D-amino acids in the brain have been elucidated. For example, D-serine (D-Ser) is an endogenous ligand for the $N$-methyl-D-aspartate receptor $(\mathrm{NMDA}-\mathrm{R})^{(17)}$. In addition, D-aspartic acid (D-Asp) ${ }^{(18)}$ and D-proline (D-Pro) ${ }^{(19)}$ injected into the lateral ventricle of neonatal chicks were found to have sedative effects, decreasing the number of distress vocalisations as well as the amount of time spent in active wakefulness.

Abbreviations: Ala, alanine; Arg, arginine; Asp, aspartic acid; GABA, $\gamma$-aminobutyric acid; GF, germ free; Gln, glutamine; His, histidine; Ile, isoleucine; Leu, leucine; Phe, phenylalanine; SPF, specific pathogen free; Ser, serine; Tau, taurine; Tyr, tyrosine; UPLC, ultra-performance liquid chromatography; Val, valine.

* Corresponding author: M. Furuse, fax +81 92642 2954, email furuse@brs.kyushu-u.ac.jp 
The L- and D-forms of amino acids have similar effects on these stress behaviours, but D-Pro ${ }^{(19)}$ and D-Asp ${ }^{(20)}$ attenuated isolation-induced stress behaviour through different mechanisms. Thus, it has been suggested that D-amino acids in vivo may have some effect on regulation of animal behaviour and that they act in synergy with L-amino acids. However, information on the relationship between intestinal microbiota and amino acid metabolism of the host brain is still equivocal. For example, there is some work reporting the presence of D-amino acids in the urine, blood serum, ethanolic extract of faeces and brain of GF rats ${ }^{(21)}$. These findings seem to indicate that some of the D-amino acids present in the body are produced endogenously without the help of the gut microbiota.

In the present study, we analysed the concentration of $\mathrm{D}$ - and L-amino acids in the plasma and brain regions of specific pathogen-free (SPF) and GF mice at two different ages ( 7 and 16 weeks), and elucidated the amino acid metabolism in the host brain by the presence or absence of the gut microbiota.

\section{Methods}

\section{Animals}

GF BALB/c mice reared at the research facilities of Tokai University School of Medicine and SPF BALB/c mice purchased from CLEA Japan, Inc. were used in the present study. These mice were given ad libitum a commercial diet (CL2 purchased from CLEA Japan, Inc.). Room temperature and moisture level were kept at $22 \pm 2{ }^{\circ} \mathrm{C}$ and $70 \%$, respectively. All of the experiments were approved by the Ethics Committee for Animal Experiments of Tokai University.

\section{Experimental procedure}

Eight mice each from the GF and SPF groups were slaughtered at 7 and 16 weeks of age, during a light period, by cervical dislocation within $3 \mathrm{~h}$ after feed withdrawal. Next, blood was collected from the arteria carotis and cervical vein as well as the brainstem, cerebellum, cerebral cortex, hippocampus, hypothalamus, striatum and thalamus. The brain samples were quickly removed from the skulls and were dissected within 8 min according to Franklin \& Paxinos ${ }^{(22)}$. In brief, after removal of the hypothalamus, the brains were cut at the median line and excised in the order of the cerebellum, brainstem, thalamus, hippocampus, striatum and cerebral cortex on an iced Petri dish. Plasma was collected from blood centrifuged at $3000 \boldsymbol{g}$ for 15 min at $4^{\circ} \mathrm{C}$ (MX-307; TOMY). All samples were rapidly frozen with dry ice and stored at $-80^{\circ} \mathrm{C}$ until further use.

\section{Analysis of free amino acids}

The concentrations of free amino acids in the plasma and brain tissues were analysed by ultra-performance liquid chromatography (UPLC) using an Acquity ${ }^{\text {TM }}$ UPLC system consisting of Waters Binary Solvent Manager, Waters Sample Manager and Waters FLR Detector (Waters), as described previously ${ }^{(18)}$. The excitation and emission wavelengths for the fluorescent detection of amino acids were 350 and $450 \mathrm{~nm}$, respectively.
The system was operated with a flow rate of $0.25 \mathrm{ml} / \mathrm{min}$ at $30^{\circ} \mathrm{C}$. The UPLC gradient system $(\mathrm{A}=50 \mathrm{~mm}$ sodium acetate ( $\mathrm{pH} 5.9$ ), B = methanol) was $10-20 \% \mathrm{~B}$ over $3.2 \mathrm{~min}, 20 \% \mathrm{~B}$ for $1 \mathrm{~min}, 20-40 \%$ B over $3.6 \mathrm{~min}, 40 \% \mathrm{~B}$ for $1.2 \mathrm{~min}, 40-60 \% \mathrm{~B}$ over $3.8 \mathrm{~min}, 60 \% \mathrm{~B}$ for $1 \mathrm{~min}$ and $60-10 \% \mathrm{~B}$ over $0.01 \mathrm{~min}$. Just before the UPLC analysis took place, each sample $(10 \mu \mathrm{l})$ was transferred to a UPLC tube, and $\mathrm{N}$-acetyl-L-cysteine/o-phthaldialdehyde $(20 \mu \mathrm{l})$ and a borate buffer $(70 \mu \mathrm{l})$ were added. Samples were then left for $2 \mathrm{~min}$ in a dark room. The same method was used for the standard solutions containing sixteen L-amino acids, sixteen D-amino acids, glycine, taurine (Tau) and so on. The concentration of amino acids in the plasma was expressed as nmol $/ \mathrm{ml}$ and the concentration of amino acids in the brain was expressed as $\mathrm{pmol} / \mathrm{mg}$ of wet tissue. Plasma was filtered through Amicon ${ }^{\circledR}$ Ultra-0.5 centrifugal filter devices $10 \mathrm{~K}$ (Millipore) with centrifugation at $14000 \mathrm{~g}$ for $15 \mathrm{~min}$ at $0^{\circ} \mathrm{C}$. Brain tissue samples were homogenised in ice-cold $0.2 \mathrm{M}$ perchloric acid and the homogenate was cooled on ice for $30 \mathrm{~min}$ for deproteinisation. After $30 \mathrm{~min}$, the mixtures were centrifuged at $20000 \mathrm{~g}$ for $15 \mathrm{~min}$ at $0^{\circ} \mathrm{C}$. After centrifugation, the supernatant was filtered through a $0 \cdot 2 \mu \mathrm{m}$ filter (Millipore), and the $\mathrm{pH}$ was adjusted to approximately $7 \cdot 0$ by adding $1 \mathrm{~m}$ sodium hydroxide. All samples were stored at $-80^{\circ} \mathrm{C}$ until further use.

\section{Statistical analyses}

All data were analysed using the statistical program StatView version 5.0 (SAS Institute, 1998). Statistical significance was evaluated using a two-way ANOVA and the Tukey-Kramer method with the Thompson outlier test. Statistical criteria for significant differences were set at $P<0.05$ for the two-way ANOVA and $P<0.01$ for the outlier test. To evaluate the brain region-specific effects on each amino acid, $q$ values were calculated using $P$ values derived from seven different brain regions in each factor, such as the microbial environment, age and interaction. BenjaminiHochberg multiple testing corrections were used for $q$ values (adjusting $P$ values), and significance was set at $q<0.05$.

\section{Results}

All data showing significant interactions between the microbial environment and age are shown in Fig. 1-3. The remaining data showing significant main effects were used to produce the heat maps shown in Fig. 4. The details of Fig. 3 are shown in the online Supplementary Table S1. Data in the online Supplementary Table S2 have no significant main effects or interactions.

In the plasma, the interaction between the microbial environment and age was significant at $P<0.005$ for D-Asp, $P<0.05$ for D-Ser, $P<0.05$ for L-alanine (L-Ala), $P<0.05$ for L-arginine (L-Arg), $P<0.01$ for L-glutamine (L-Gln) and $P<0 \cdot 001$ for Tau (Fig. 1(a)-(f)). The concentrations of D-Asp, L-Ala, L-Gln and Tau decreased in SPF mice from 7 to 16 weeks of age, but with the GF mice, the concentrations were constant between 7 and 16 weeks of age. A similar concentration of L-Arg was observed in both GF and SPF mice at 7 weeks of age, but a further significant increase between 7 and 16 weeks of age was observed in SPF mice only. 

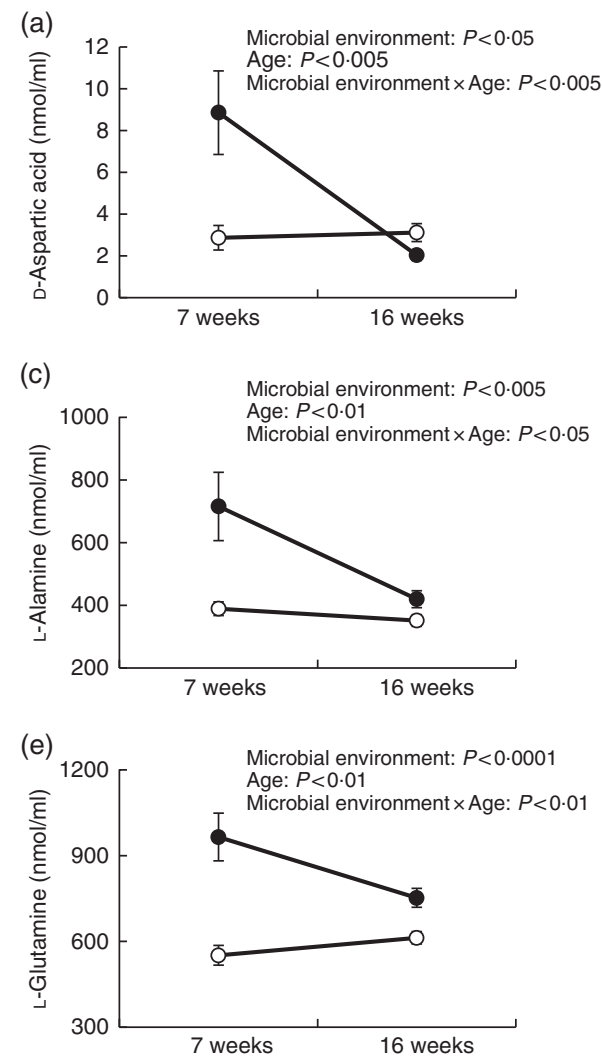
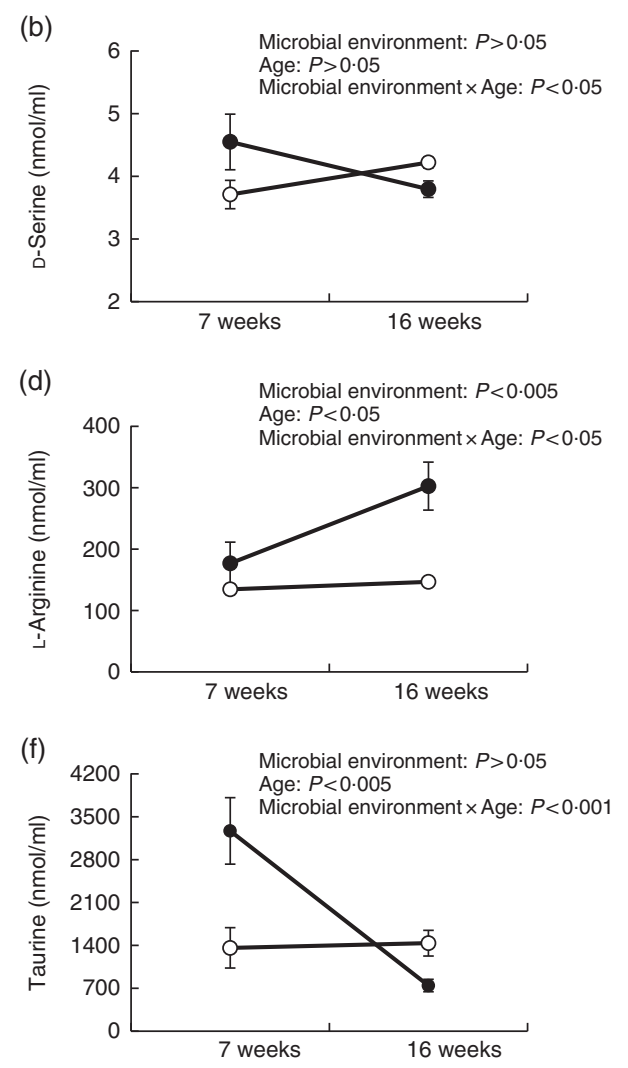

Fig. 1. The effect of microbial environment and age on concentration of amino acids in the plasma of specific pathogen-free ( --$)$ and germ-free $(-\bigcirc)$ mice (n 7-9). Values are means with standard errors for (a) D-aspartic acid, (b) D-serine, (c) L-alanine, (d) L-arginine, (e) L-glutamine and (f) taurine.

In the striatum, the interaction between the microbial environment and age was significant for L-Asp $(q<0.05)$, L-Ala $(q<0.05)$, L-Arg $(q<0.01)$, L-valine (L-Val) $(q<0.05)$ and Tau $(q<0.05)$ (Fig. 2(a)-(e)). The concentrations of L-Asp, L-Ala, L-Arg and L-Val were high at 7 weeks of age in SPF mice and decreased toward 16 weeks of age, whereas in GF mice the concentrations remained low and constant over the same period.

In the cerebellum, the interaction between the microbial environment and age was significant for D-Asp $(q<0.05)$, L-Asp $(q<0.05)$, L-Ala $(q<0.005)$ and Tau $(q<0.001)$ (Fig. 3(a)-(d)). The concentration of D-Asp at 7 weeks of age was almost identical in both the groups of mice, but toward 16 weeks of age the concentration greatly decreased in SPF mice. At 7 weeks of age, the concentration of L-Asp was higher in SPF mice than in GF mice, but that in GF mice steadily increased over time to eventually become similar at 16 weeks of age. At 7 weeks of age, the concentration of L-Ala was higher in SPF mice than it was in GF mice but dropped at 16 weeks of age, whereas over the same period it remained constant in GF mice. The concentration of Tau was higher in SPF mice than it was in GF mice at 7 weeks of age, but the opposite was true at 16 weeks of age. In the cerebral cortex, the interaction between the microbial environment and age was significant for L-histidine (L-His) $(q<0.05)$ (Fig. 3(e)). As they aged, both SPF and GF mice had a lower concentration of L-His, but the decrease was greater in GF mice than it was in SPF mice. In the hippocampus, the interaction between the microbial environment and age were significant in L-Asp $(q<0.05)$ (Fig. 3(f)). The concentration of L-Asp was higher in SPF mice than it was in GF mice, but the concentration decreased in all mice as they aged. There were no differences in L-Asp concentration between GF and SPF mice at 16 weeks of age.

The mean values of amino acid concentrations without significant interactions were used to produce heat maps (Fig. 4).

Regarding the main effect of gut microbiota, SPF mice had significantly higher concentrations of L-Asp $(P<0 \cdot 01)$, D-Ala $(P<0.0001)$, L-His $(P<0.05)$, L-isoleucine (L-Ile) $(P<0.01)$, L-leucine (L-Leu) $\quad(P<0.0001), \quad$ L-phenylalanine (L-Phe) $(P<0.0005)$ and $\mathrm{L}-\mathrm{Val}(P<0.001)$ in plasma than did GF mice (Fig. 4(a)). D-Ala could not be detected in GF mice. In the plasma, the concentrations of L-tyrosine (L-Tyr) $(P<0 \cdot 005)$, L-Ile $(P<0.0005)$, L-Leu $(P<0.0005)$, L-Phe $(P<0.0005)$ and $\mathrm{L}-\mathrm{Val}$ $(P<0.0005)$ increased with age (Fig. $4($ a) ).

In the brainstem, the concentrations of L-Arg $(q<0.0005)$, L-Ala $(q<0.05)$ and L-Gln $(q<0.05)$ were higher in the SPF mice than those in the GF mice, whereas the opposite was true for the concentrations of D-Asp $(q<0.001)$ and L-Ser $(q<0.001)$ (Fig. 4(b)). As mice aged, the concentrations of L-Ser $(q<0.001)$, L-Ala $(q<0.05)$, L-Ile $(q<0 \cdot 001)$ and L-Leu $(P<0 \cdot 0005)$ elevated, whereas that of $\gamma$-aminobutyric acid (GABA) slightly decreased.

In the cerebellum, the concentrations of L-Gln $(q<0.0005)$, L-Ile $(q<0.001)$, L-Leu $(q<0.0005)$, L-Phe $(q<0.005)$, GABA $(q<0.05)$ and L-Val $(q<0.01)$ were significantly higher in the 
(a)

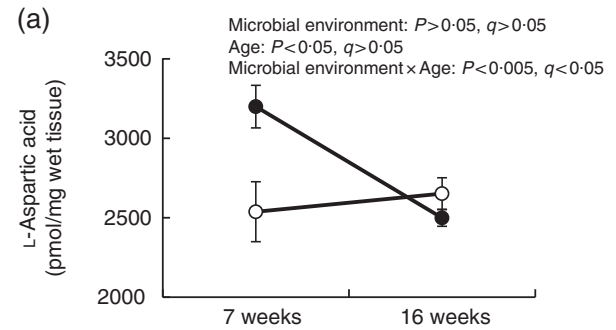

(c) Microbial environment: $P<0.0005, q<0.0005$

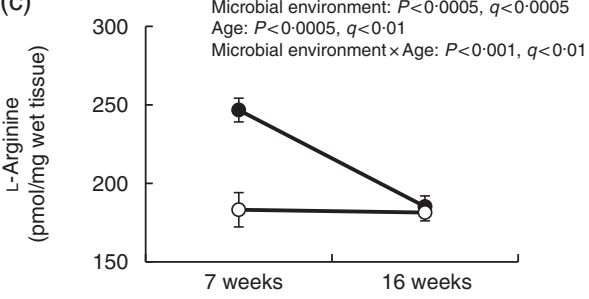

(b) Microbial environment: $P>0.05, q>0.05$

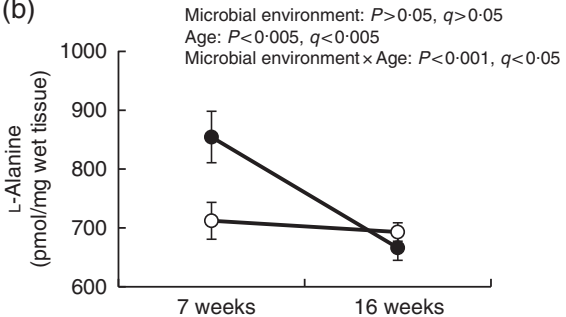

(d) Microbial environment: $P>0.05, q>0.05$

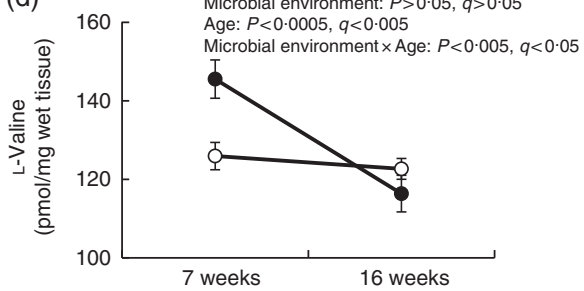

(e) Microbial environment: $P>0.05, q>0.05$

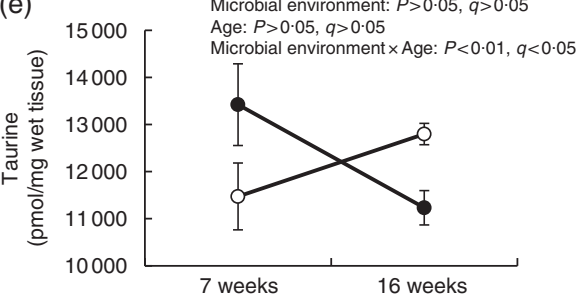

Fig. 2. The effect of microbial environment and age on amino acid concentrations in the striatum of specific pathogen-free ( --$)$ and germ-free ( $-\bigcirc)$ mice $(n 7-9)$. Values are means with standard errors for (a) L-aspartic acid, (b) L-alanine, (c) L-arginine, (d) L-valine and (e) taurine.

SPF mice than those in the GF mice (Fig. 4(c)). The concentration of L-Ile $(q<0.0005)$ increased as the mice aged.

In the cerebral cortex, the concentrations of L-Arg $(q<0 \cdot 005)$, L-Gln $(q<0 \cdot 0005)$ and L-Leu $(q<0.05)$ were significantly higher in the SPF mice than those in the GF mice, but the opposite was true for the concentrations of D-Ser $(q<0.001)$ and L-Ser $(q<0.005)$ (Fig. 4(d)). The concentrations of D-Asp $(q<0.0005)$, D-Ser $(q<0.0005)$, L-Ser $(q<0.0005)$, L-Ala $(q<0.0005)$, L-Leu $(q<0.0005)$ and Tau $(q<0.0005)$ increased as the mice aged, but the opposite was true for the concentrations of L-Arg $(q<0.0005)$, L-Val $(q<0.05)$ and GABA $(q<0.001)$

In the hippocampus, the concentrations of L-Ala $(q<0.005)$, L-Arg $(q<0.0005)$, L-Gln $(q<0.0005)$, L-Ile $(q<0.01)$, L-Leu $(q<0.0005)$, L-Phe $(q<0.005)$, L-Val $(q<0.05)$ and GABA $(q<0.05)$ were significantly higher in the SPF mice than those in the GF mice (Fig. 4(e)). Except for that of L-Leu $(q<0 \cdot 01)$, which increased, the concentrations of D-Asp $(q<0 \cdot 0005)$, L-Ala $(q<0.0005)$, L-Arg $(q<0.0005)$, L-His $(q<0.05)$, L-Ile $(q<0.005)$, L-Phe $(q<0.0005)$, L-Val $(q<0 \cdot 001)$, GABA $(q<0.05)$ and Tau $(q<0.0005)$ decreased as the mice aged.

In the hypothalamus, the concentrations of L-Arg $(q<0.0005)$, L-Gln $(q<0.0005)$, L-Ile $(q<0.01)$, L-Leu $(q<0.0005)$ and L-Phe $(q<0.005)$ were significantly higher in the SPF mice than those in the GF mice (Fig. 4(f)). As the mice aged, the concentrations of D-Asp $(q<0.05)$ and L-Ala $(q<0.005)$ decreased, but L-Leu $(q<0.0005)$ increased.

In the striatum, the concentrations of L-Leu $(q<0.0005)$, L-Ile $(q<0.05)$ and L-Gln $(q<0.0005)$ were significantly higher in the SPF mice than those in the GF mice (Fig. $4(\mathrm{~g})$ ). The concentrations of L-Tyr $(P<0.005)$, L-Phe $(P<0.01)$ and L-Ile $(q<0.05)$ decreased as the mice aged.

In the thalamus, the concentrations of L-Arg $(q<0.0005)$, L-Gln $(q<0.0005)$ and L-Leu $(q<0.005)$ were significantly higher in the SPF mice than in the GF mice, whereas the opposite was true for the concentrations of D-Asp $(q<0.05)$, D-Ser $(q<0.0005)$ and L-Ser $(q<0.0005)$ (Fig. 4(h)). The concentrations of L-Ser $(q<0.05)$ and L-Leu $(q<0.0005)$ increased as the mice aged, whereas that of L-His $(q<0.005)$ decreased. The weight of the brains of the SPF and GF mice at different ages is shown in the online Supplementary Fig. S1. The interaction between the microbial environment and age was significant $(P<0 \cdot 001)$, implying that the brain weight of SPF mice increased with age, but that of GF mice remained constant.

The concentration of D-amino acid as a proportion of the total $\mathrm{D}$ - and L-amino acid concentration is shown in the online Supplementary Fig. S2 and S3. The interaction between the microbial environment and age for Asp was significant in the plasma $(P<0.0001)$, hippocampus $(P<0.005)$, striatum $(P<0.05)$ and thalamus $(P<0.05)$ (online Supplementary Fig. S2). The proportion of $\mathrm{D}$-Asp in the plasma decreased with age in SPF mice, but the proportion increased in the cerebellum, cerebral cortex and hypothalamus with age. Regarding the main effect of the gut microbiota, the proportion of Asp in the brainstem $(P<0.0001)$, cerebellum $(P<0.05)$, cerebral cortex $(P<0.0001)$ and the hypothalamus $(P<0.05)$ of SPF mice was significantly lower than it was in GF mice 
(a)

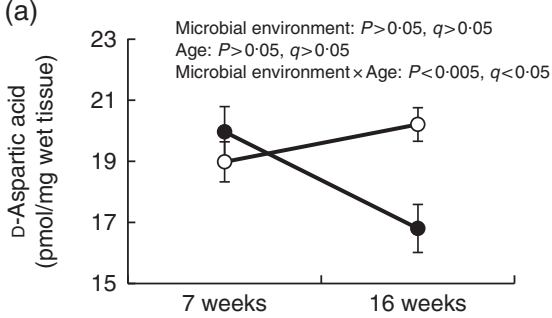

(c) Microbial environment: $P<0.0001, q<0.001$

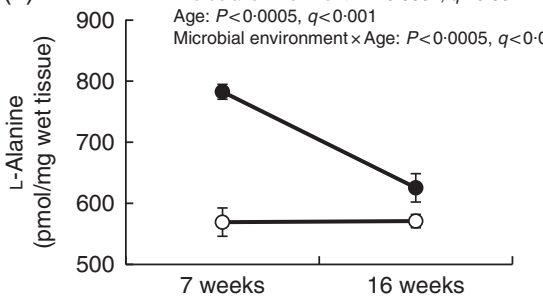

(e) Microbial environment: $P>0.05, q>0.05$

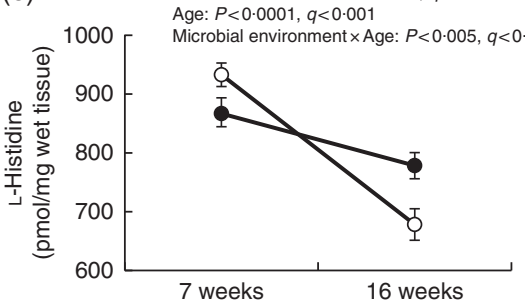

(b)

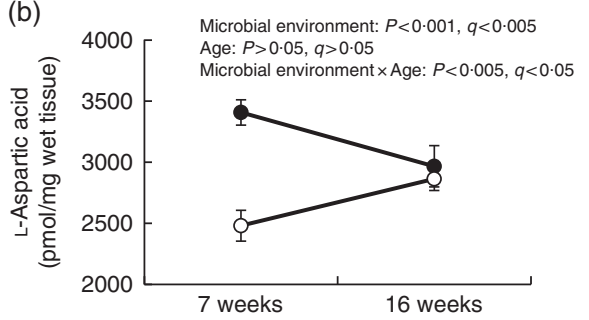

(d) Microbial environment: $P>0.05, q>0.05$

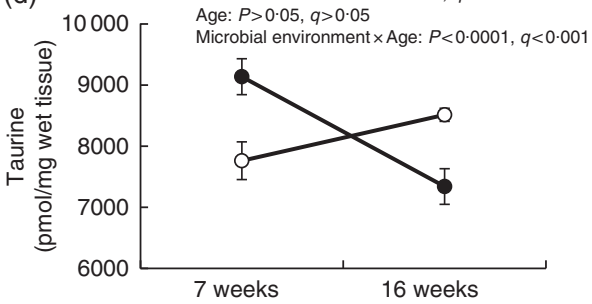

Microbial environment: $P<0.0005, q<0.005$

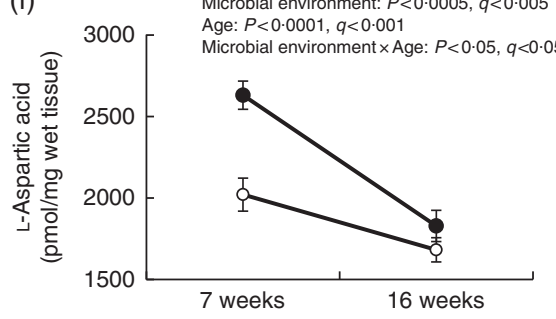

Fig. 3. The effect of microbial environment and age on amino acid concentrations in the cerebellum, cerebral cortex and hippocampus of specific pathogen-free (- - ) and germ-free (-O) mice (n 7-9). Values are means with standard errors for (a) D-aspartic acid, (b) L-aspartic acid, (c) L-alanine, (d) taurine in the cerebellum, (e) L-histidine in the cerebral cortex and (f) L-aspartic acid in the hippocampus.

(online Supplementary Table S2). The proportion of Asp decreased in the cerebellum $(P<0 \cdot 0001)$ and hypothalamus $(P<0.0001)$ with age, but increased in the cerebral cortex $(P<0.0001)$. The differences in the proportions of $\mathrm{D}-$ and $\mathrm{L}-\mathrm{Ser}$ between the GF and SPF mice increased in the plasma as the mice aged $(P<0.05)$, but in the striatum, although there was a large difference at 7 weeks of age, they decreased as the mice aged $(P<0.05)$ (online Supplementary Fig. S3). The SPF mice had a higher proportion of Ser in the brainstem than did the GF mice $(P<0.05)$. In contrast, the GF mice had a higher proportion of Ser in the cerebral cortex $(P<0 \cdot 01)$, hippocampus $(P<0 \cdot 0001)$ and thalamus $(P<0.005)$, than did the SPF mice (online Supplementary online Supplementary Table S4). In general, the proportion of Ser increased in the cerebral cortex $(P<0.05)$, but decreased in the thalamus $(P<0 \cdot 0001)$, as the mice aged.

\section{Discussion}

Several differences were observed between SPF and GF mice in terms of fluctuating plasma amino acid concentrations. Gut microbiota influences secretions, promotes digestion and absorption, and stimulates the immunological system and HPA axis of the host. The results of the present study indicated that the presence of gut microbiota is largely associated with amino acid concentration in the blood and brain of the host. Although the mechanism of this phenomenon remains unclear, it may be possible that either (1) gut microbiota modifies the amino acid metabolism and/or breaks down amino acids in the gut, and modified amino acids are then absorbed, (2) the physiological activity of the constitutive substances of microbiota such as proteins or peptides affect the amino acid metabolism or (3) both instances occur. Although a detailed study of the intestinal amino acid metabolism was beyond the scope of the present work, some conclusions can be drawn from the correlation found between the D-amino acid metabolism and the presence of gut microbiota. For example, the peptide D-Ala-D-Ser was found to constitute the bacterial cell walls ${ }^{(23)}$, and Csapó et al. ${ }^{(24)}$ previously suggested that D-Asp and D-glutamic acid (D-Glu) likely were bacterial protein markers. In this study, D-Ala was detected in the plasma of SPF mice, but not in that of GF mice, which might indicate that our first hypothesis about the modification or breakdown of amino acids by the gut microbiota and subsequent absorption is correct. Furthermore, Konno et $a l^{(25)}$ found that the urine of GF mice had a lower D-Ala concentration than did that of conventional mice. These workers suggested that D-Ala was derived from the cell wall of the gut microbiota. Furthermore, Kleckner \& Dingledine ${ }^{(26)}$ remarked that D-Ala was enabled to activate NMDA-R, which implicated brain functions through the transmission of excitatory signal ${ }^{(27-29)}$. However, in our study no D-Ala was detected in the analysed brain regions. Even though the gut microbiota may be involved in the production of D-Ala, the concentration is too low to reflect an amount of D-Ala in the brain. Nonetheless, Nagata et al. ${ }^{(30)}$ reported that when $0.5 \%$ D-Ala was added to 
(a)

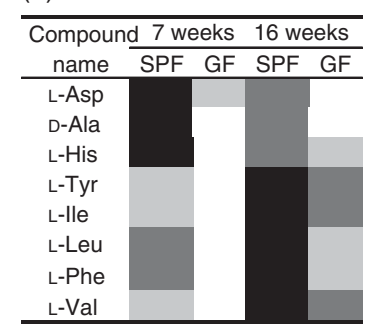

(d)

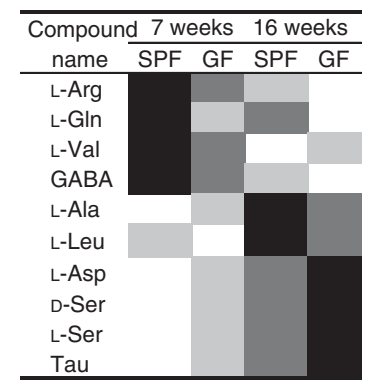

(g)

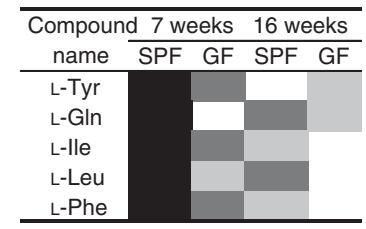

(b)

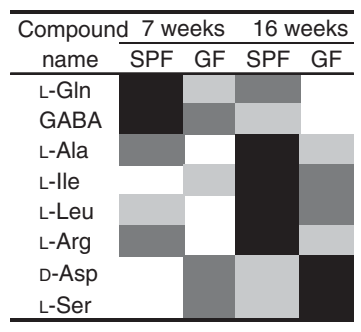

(e)

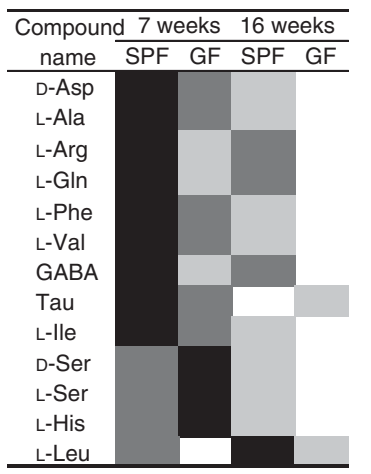

(h)

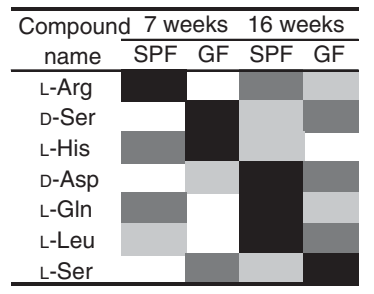

(c)

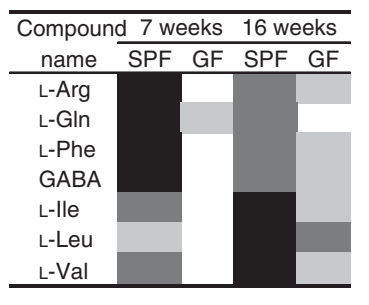

(f)

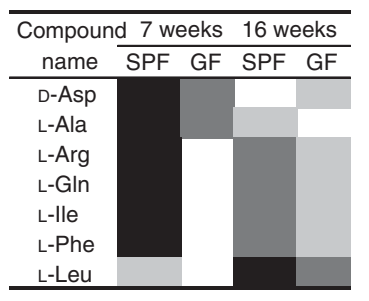

Fig. 4. Heat maps of the converted mean values of the concentration of amino acids. Mean values are shown in the online Supplementary Table S1. (a) Plasma, (b) brainstem, (c) cerebellum, (d) cerebral cortex, (e) hippocampus, (f) hypothalamus, (g) striatum and (h) thalamus. The colour depth shows values from high to low in decreasing order, as $\square, \square, \square$ and $\square$. SPF, specific pathogen free; GF, germ free; Asp, aspartic acid; Ala, alanine; His, histidine; Tyr, tyrosine; lle, isoleucine; Leu, leucine; Phe, phenylalanine; Val, valine; Gln, glutamine; GABA, $\gamma$-aminobutyric acid; Arg, arginine; Ser, serine; Tau, taurine.

drinking water and orally administered to mutant mice lacking D-amino acid oxidase for 2 weeks, the concentration of D-Ala in the brain greatly increased. In the case of normally D-amino acid oxidase-producing mice, brain D-Ala also increased, but to a lesser extent. In the work by Nagata et al., administration of $0.5 \% \mathrm{D}-\mathrm{Ala}$ in drinking water caused the concentration of serum D-Ala in mice to be $34.2 \mathrm{nmol} / \mathrm{ml}$. This previously reported concentration was three times higher than that obtained in the present study. The reason for the negligible amount of D-Ala in the brain found in the present study may be a low D-Ala production in the gut of SPF mice.

It can be suggested that mice endogenously produced D-Ser and that as they aged, the gut microbiota and/or their products inhibited the production of D-Ser. This is believed to have occurred because the concentration of L-Ser, substrate of D-Ser and the amount of D-Ser as a proportion of the total amount of D- and L-Ser - one of the indicators of the activity of serine racemase - did not differ between SPF and GF mice at 7 weeks of age. However, at 16 weeks of age, D-Ser concentration in the plasma of SPF mice was lower than it was in GF mice, although L-Ser concentration did not differ between the SPF and GF mice.
However, at 16 weeks of age, the amount of D-Ser, as a proportion of the total amount of D- and L-Ser, was higher in the GF mice than in the SPF mice.

Regarding D-Ser in the brain, we successfully determined its concentration in all the brain regions except for the cerebellum. Interestingly, the concentration of D-Ser between SPF and GF mice, as well as between the plasma and brain regions, was found to be different. For example, although no differences in the concentration of D-Ser were found in the plasma SPF and GF mice at 7 weeks of age, the concentration of D-Ser in some brain regions was higher in GF mice than in SPF mice. These differences suggest that the presence of gut microbiota may dictate microbial products, vagus nerves, cytokines, or the HPA axis to regulate the activity of serine racemase in the host brain $^{(6,31,32)}$. Nonetheless, in the present study, D-Ser was hardly detected in the cerebellum. Morikawa et $a l^{(33)}$ previously reported that $\mathrm{D}$-Ser concentration was very low in the cerebellum of mice. D-Ser is an endogenous ligand for NMDA-R, itself associated with neurological diseases involving synaptic plasticity or neuronal degeneration ${ }^{(34-36)}$. In the cerebral cortex and the thalamus, D-Ser concentration was lower in SPF mice 
than it was in GF mice. The cerebral cortex has some functional areas. For example, the intraparietal cortex and the superior frontal cortex are involved in preparing for and applying goal-directed selections to stimuli and responses ${ }^{(37)}$. In addition, the thalamus has many roles in brain-networking and Parkinson's disease ${ }^{(38-40)}$. In this context, we theorise that the gut microbiota has the potential to regulate neurological diseases by altering D-Ser metabolism in the host brain. This hypothesis seems to be supported by changes detected in D-Ser metabolism, possibly due to L-Ser synthesis being more active in GF mice than in SPF mice. Higher concentrations of L-Ser and D-Ser in the cerebral cortex and thalamus of GF mice than in those of SPF mice also seem to corroborate our hypothesis. Furthermore, in the brain, L-Ser is synthesised from glucose or produced by proteolysis ${ }^{(41)}$, which also seems to suggest that gut microbiota regulates L-Ser biosynthesis.

L-Tyr is metabolised to catecholamines such as DA, NA and adrenaline $^{(42)}$, and in the brainstem, catecholaminergic neurons are known to exist ${ }^{(43)}$. The GF mice had higher concentrations of DA and NA in the brainstem and were more active than mice carrying commensal faecal microbiota upon inoculation with gut microbiota $^{(9)}$. In this study, the concentration of L-Tyr in the plasma and brain regions did not differ between SPF and GF mice. Thus, it was determined that the gut microbiota had no influence on L-Tyr absorption or synthesis, although it may still regulate the metabolism of tyrosine to catecholamine.

GABA is an inhibitory neurotransmitter in the $\mathrm{CNS}^{(44)}$. In this study, the concentration of GABA was higher in the cerebellum and hippocampus of SPF mice than it was in those of GF mice. The cerebellum is involved not only in motor regulation but also in cognitive functions including attention, memory and learning tasks in humans ${ }^{(45)}$. It is well known that the hippocampus is involved in information acquisition and retention, and neurogenesis ${ }^{(46,47)}$. $\mathrm{L}$-Glu is also believed to be a neurotransmitter and is metabolised to GABA by glutamate decarboxylase ${ }^{(48)}$. In the present study, although L-Glu was not analysed, the concentration of the reversible metabolite $\mathrm{L}-\mathrm{Gln}$ was higher in the plasma, brainstem, cerebellum, cerebral cortex, hippocampus, hypothalamus, striatum and thalamus of SPF mice than it was in those of GF mice. As a result, it is believed that the gut microbiota may play a role in facilitating the metabolism of L-Gln. Differences in the concentrations of GABA and $\mathrm{L}-\mathrm{Gln}$ between SPF and GF mice may exert an effect on the functions of these brain regions.

L-Ile, L-Leu and L-Val are commonly known as branchedchain amino acids (BCAA). BCAA are not only used as substrates for protein, but also used to promote protein synthesis via activation of the mammalian target of rapamycin $(\mathrm{mTOR})^{(49)}$. In particular, L-Leu strongly affects mTOR activation. In this study, the concentration of L-Leu in the plasma and most brain regions of SPF mice was higher than it was in those of GF mice. The gut microbiota may assist in absorption of L-Leu in intestinal epithelial cells and also intervene in L-Leu metabolism. Hence, there is a possibility that the gut microbiota is involved in host protein synthesis in the periphery or CNS.

L-His is metabolised to histamine, which modulates glutamate NMDA-R and is involved in several psychological states including cognative arousal, anxiety and eating suppression ${ }^{(50)}$. In the present study, the concentration of L-His in the cerebral cortex decreased as the mice aged. Furthermore, although at 7 weeks of age the concentration of L-His in the cerebral cortex of SPF mice was lower than it was in that of GF mice, the difference was reversed at 16 weeks of age. It is believed that the gut microbiota could affect the central histaminergic system via the changing metabolism of L-His. Therefore, it can be suggested that L-His metabolism changes with aging, but this alteration can be alleviated by the activity of the gut microbiota.

In the present study, we were especially interested in the changes in the concentration of free amino acid in some brain regions. A previous study at these facilities confirmed that changes in the concentration of free amino acid occurred in the brains of neonatal chicks exposed to either restraint and isolation-induced or fasting stress. The concentration of L-Pro and L-Arg was found to decrease in the telencephalon and diencephalon under stress ${ }^{(51)}$, but an intracerebroventricular injection of L-Pro, L-Arg and several other amino acids exerted a sedative or hypnotic effect on animals under an acute stress ${ }^{(13)}$. In a separate study, it was investigated whether depression-like states affected amino acid metabolism and the potential roles of amino acid metabolism on stressregulatory mechanisms, by comparing Wistar-Kyoto rats, an animal model of depression-like behaviour and stress vulnerability, with control Wistar rats. The metabolism of amino acids in brain tissues of Wistar-Kyoto rats was greatly different from that of normal Wistar rats. This previous report seems to indicate that an abnormal amino acid metabolism may induce depression-like behaviour and stress vulnerability in Wistar-Kyoto rats ${ }^{(52)}$.

In conclusion, the activity of microbiota present in the gut seems to change the concentration of $\mathrm{D}$ - and $\mathrm{L}$-amino acids in the plasma and some brain regions of mice, and thus, the gut microbiota may be an important regulator of amino acid metabolism. Further research is warranted to clarify the role of the gut microbiota in the metabolism of amino acids. Hence, it is foreseeable that future research may involve transplant of specific microbial strains or inoculation of antibiotic substances to manipulate microbiota communities, which may be used to evaluate the mental health and cognitive abilities of humans, including mood disorders and problem-solving tasks, respectively.

\section{Acknowledgements}

The authors thank Yuji Aiba for his technical assistance.

There was no financial support.

M. F. conceived and designed the study. T. K., M. N. and H. I. performed the experiments and conducted the data analysis. T. K., S. Y., Y. K. and M. F. wrote the manuscript.

The authors declare that there are no conflicts of interest.

\section{Supplementary material}

For supplementary material/s referred to in this article, please visit https://doi.org/10.1017/S0007114517000678 


\section{References}

1. Bäckhed F, Ley RE, Sonnenburg JL, et al. (2005) Host-bacterial mutualism in the human intestine. Science 307, 1915-1920.

2. Fava F \& Danese S (2011) Intestinal microbiota in inflammatory bowel disease: friend or foe? World J Gastroenterol 17, 557-566.

3. Finlay BB (1990) Cell adhesion and invasion mechanisms in microbial pathogenesis. Curr Opin Cell Biol 2, 815-820.

4. Sudo N, Sawamura SA, Tanaka K, et al. (1997) The requirement of intestinal bacterial flora for the development of an IgE production system fully susceptible to oral tolerance induction. I Immunol 159, 1739-1745.

5. Sudo N, Chida Y, Aiba Y, et al. (2004) Postnatal microbial colonization programs the hypothalamic-pituitary-adrenal system for stress response in mice. J Physiol 558, 263-275.

6. Cryan JF \& Dinan TG (2012) Mind-altering microorganisms: the impact of the gut microbiota on brain and behavior. Nat Rev Neurosci 13, 701-712.

7. Collins SM, Surette M \& Bercik P (2012) The interplay between the intestinal microbiota and the brain. Nat Rev Microbiol 10, $735-742$.

8. Neufeld KM, Kang N, Bienenstock J, et al. (2011) Reduced anxiety-like behavior and central neurochemical change in germ-free mice. Neurogastroenterol Motil 23, 255-264.

9. Nishino R, Mikami K, Takahashi H, et al. (2013) Commensal microbiota modulate murine behaviors in a strictly contamination-free environment confirmed by culture-based methods. Neurogastroenterol Motil 25, 521-528.

10. Adams JB, Johansen LJ, Powell LD, et al. (2011) Gastrointestinal flora and gastrointestinal status in children with autism - comparisons to typical children and correlation with autism severity. BMC Gastroenterol 11, 22.

11. Wang Y \& Kasper LH (2014) The role of microbiome in central nervous system disorders. Brain Behav Immun 38, 1-12.

12. Naseribafrouei A, Hestad K, Avershina E, et al. (2014) Correlation between the human fecal microbiota and depression. Neurogastroenterol Motil 26, 1155-1162.

13. Furuse $M$ (2015) Screening of central functions of amino acids and their metabolites for sedative and hypnotic effects using chick model. Eur J Pharmacol 762, 382-393.

14. Wolosker H, Blackshaw S \& Snyder SH (1999) Serine racemase: a glial enzyme synthesizing D-serine to regulate glutamate-N-methyl-D-aspartate neurotransmission. Proc Natl Acad Sci U S A 96, 13409-13414.

15. Stevens ER, Esguerra M, Kim PM, et al. (2003) D-serine and serine racemase are present in the vertebrate retina and contribute to the physiological activation of NMDA receptors. Proc Natl Acad Sci U S A 100, 6789-6794.

16. Lam H, Oh DC, Cava F, et al. (2009) D-amino acids govern stationary phase cell wall remodeling in bacteria. Science $\mathbf{3 2 5}$, $1552-1555$.

17. Mothet JP, Parent AT, Wolosker H, et al. (2000) D-serine is an endogenous ligand for the glycine site of the N-methylD-aspartate receptor. Proc Natl Acad Sci U S A 97, 4926-4931.

18. Erwan E, Tomonaga S, Ohmori T, et al. (2013) Oral administration of D-aspartate, but not of L-aspartate, reduces food intake in chicks. J Poult Sci 50, 165-171.

19. Hamasu K, Shigemi K, Tsuneyoshi Y, et al. (2010) Intracerebroventricular injection of L-proline and D-proline induces sedative and hypnotic effects by different mechanisms under an acute stressful condition in chicks. Amino Acids 38, 57-64.

20. Erwan E, Chowdhury VS, Nagasawa M, et al. (2014) Central injection of $\mathrm{L}^{-}$and $\mathrm{D}$-aspartate attenuates isolation induced stress behavior in chicks possibly through different mechanisms. Eur J Pharmacol 736, 138-142.
21. Brückner H \& Schieber A (2001) Ascertainment of D-amino acids in germ-free, gnotobiotic and normal laboratory rats. Biomed Chromatogr 15, 257-262.

22. Franklin KBJ \& Paxinos G (2007) The Mouse Brain in Stereotaxic Coordinates, 3rd ed. New York: Academic Press.

23. Arias CA, Weisner J, Blackburn JM, et al. (2000) Serine and alanine racemase activities of VanT: a protein necessary for vancomycin resistance in Enterococcus gallinarum BM4174. Microbiology 146, 1727-1734.

24. Csapó J, Schmidt J, Csapó-Kiss Z, et al. (2001) A new method for the quantitative determination of protein of bacterial origin on the basis of D-aspartic acid and D-glutamic acid content. Acta Aliment 30, 37-52.

25. Konno R, Oowada T, Ozaki A, et al. (1993) Origin of D-alanine present in urine of mutant mice lacking D-amino-acid oxidase activity. Am J Physiol 265, G699-G703.

26. Kleckner NW \& Dingledine R (1988) Requirement for glycine in activation of NMDA-receptors expressed in Xenopus oocytes. Science 241, 835-837.

27. Nakanishi S (1992) Molecular diversity of glutamate receptors and implications for brain function. Science $\mathbf{2 5 8}$, 597-603.

28. Monyer H, Burnashev N, Laurie DJ, et al. (1994) Developmental and regional expression in the rat brain and functional properties of four NMDA receptors. Neuron 12, 529-540.

29. McEwen BS (1999) Stress and hippocampal plasticity. Annu Rev Neurosci 22, 105-122.

30. Nagata Y, Konno R \& Niwa A (1994) Amino acid levels in D-alanine-administered mutant mice lacking D-amino acid oxidase. Metabolism 43, 1153-1157.

31. Bravo JA, Forsythe P, Chew MV, et al. (2011) Ingestion of Lactobacillus strain regulates emotional behavior and central GABA receptor expression in a mouse via the vagus nerve. Proc Nat Acad Sci U S A 108, 16050-16055.

32. Ait-Belgnaoui A, Durand H, Cartier C, et al. (2012) Prevention of gut leakiness by a probiotic treatment leads to attenuated HPA response to an acute psychological stress in rats. Psychoneuroendocrinology 37, 1885-1895.

33. Morikawa A, Hamase K, Inoue T, et al. (2007) Alterations in D-amino acid levels in the brains of mice and rats after the administration of D-amino acids. Amino Acids 32, 13-20.

34. Jenrsch JD \& Roth RH (1999) The neuropsychopharmacology of phencyclidine: from NMDA receptor hypofunction to the dopamine hypothesis of schizophrenia. Neuropsychopharmacology 20, 201-225.

35. Zeron MM, Hansson O, Chen N, et al. (2002) Increased sensitivity to N-methyl-D-aspartate receptor-mediated excitotoxicity in a mouse model of Huntington's disease. Neuron 33, 849-860.

36. Liu L, Wong TP, Pozza MF, et al. (2004) Role of NMDA receptor subtypes in governing the direction of hippocampal synaptic plasticity. Science 304, 1021-1024.

37. Corbetta M \& Shulman GL (2002) Control of goal-directed and stimulus-driven attention in the brain. Nat Rev Neurosci 3, 201-215.

38. Benabid AL, Pollak P, Gao D, et al. (1996) Chronic electrical stimulation of the ventralis intermedius nucleus of the thalamus as a treatment of movement disorders. J Neurosurg $\mathbf{8 4}$, 203-214.

39. Apkarian AV, Bushnell MC, Treede RD, et al. (2005) Human brain mechanisms of pain perception and regulation in health and disease. Eur J Pain 9, 463-484.

40. Cameron IGM \& Watanabe M (2010) Differential contributions of thalamic nuclei in the generation of antisaccades. J Neurophysiol 104, 2306-2307. 
41. Verleysdonk S \& Hamprecht B (2000) Synthesis and release of L-serine by rat astroglia-rich primary cultures. Glia 30, 19-26.

42. Zhou QY, Quaife CJ \& Palmiter RD (1995) Targeted disruption of the tyrosine hydroxylase gene reveals that catecholamines are required for mouse fetal development. Nature 374, 640-643.

43. LaVoie MJ, Ostaszewski BL, Weihofen A, et al. (2005) Dopamine covalently modifies and functionally inactivates parkin. Nat Med 11, 1214-1221.

44. Johnson JW \& Ascher P (1987) Glycine potentiates the NMDA response in cultured mouse brain neurons. Nature $\mathbf{3 2 5}$ 529-531.

45. Timmann D \& Daum I (2007) Cerebellar contributions to cognitive functions: a progress report after two decades of research. Cerebellum 6, 159-162.

46. Moser MB \& Moser EI (1998) Functional differentiation in the hippocampus. Hippocampus 8, 608-619.
47. Shors TJ, Townsend DA, Zhao M, et al. (2002) Neurogenesis may relate to some but not all types of hippocampaldependent learning. Hippocampus 12, 578-584.

48. Danbolt NC (2001) Glutamate uptake. Prog Neurobiol 65, 1-105.

49. Mattick JS, Kamisoglu K, Ierapetritou MG, et al. (2013) Branchedchain amino acid supplementation: impact on signaling and relevance to critical illness. Wiley Interdiscip Rev Syst Biol Med $\mathbf{5}$, $449-460$

50. Brown RE, Stevens DR \& Haas HL (2001) The physiology of brain histamine. Prog Neurobiol 63, 637-672.

51. Hamasu K, Haraguchi T, Kabuki Y, et al. (2009) L-proline is a sedative regulator of acute stress in the brain of neonatal chicks. Amino Acids 37, 377-382.

52. Nagasawa M, Ogino Y, Kurata K, et al. (2012) Hypothesis with abnormal amino acid metabolism in depression and stress vulnerability in Wistar Kyoto rats. Amino Acids 43, 2101-2111. 